\title{
BACTERIOLOGICAL PROFILE OF ACUTE BACTERIAL MENINGITIS IN ADULTS IN A TERTIARY CARE HOSPITAL IN NORTH KERALA
}

\author{
Irene Jose Manjiyili1 ${ }^{1}$ Anitha Puduvail Moorkoth², Rema Devi Santhakumari ${ }^{3}$
}

${ }^{1}$ Assistant Professor, Department of Microbiology, Government Medical College, Thrissur, Kerala.

${ }^{2}$ Associate Professor, Department of Microbiology, Government Medical College, Kozhikode, Kerala.

${ }^{3}$ Professor and HOD, Department of Microbiology, Government Medical College, Thiruvananthapuram, Kerala.

\section{ABSTRACT}

\section{CONTEXT}

Acute Bacterial Meningitis (ABM) is a medical emergency requiring immediate diagnosis and treatment. The bacterial pathogens responsible for $\mathrm{ABM}$ may vary with time, geographic distribution, age and preceding medical and/or surgical conditions of the patient. Information regarding the changing trends in terms of aetiology and antibiotic susceptibility in a particular region is essential for the correct and timely management of ABM.

\section{AIM}

This study was done to determine the bacterial pathogens responsible for ABM in adults and study their antibiotic susceptibility pattern.

\section{SETTINGS AND DESIGN}

A cross-sectional study was carried out on clinically suspected cases of ABM at Government Medical College, Kozhikode, for a period of one year.

\section{METHODS AND MATERIALS}

Samples of Cerebrospinal Fluid (CSF) collected aseptically from clinically suspected cases of ABM were centrifuged and subjected to culture, Gram staining and antigen detection by Latex Agglutination Test (LAT). Antibiotic susceptibility testing was performed on all the isolates. Cases of post-traumatic meningitis and meningitis developing after neurosurgical procedures were also included in the study.

\section{RESULTS}

CSF samples were collected from 165 clinically suspected cases of ABM and processed. The bacterial pathogen could be identified by centrifuged Gram stain in 21 cases (12.7\%), by culture in 16 cases (9.7\%) and by LAT in nine cases (5.4\%). Streptococcus pneumoniae was the predominant pathogen isolated in 7 cases (43.8\%) followed by Pseudomonas aeruginosa in 4 cases (25\%), Acinetobacter baumannii and Coagulase negative staphylococci (CoNS) in two cases each (12.5\%) and Staphylococcus aureus in one case (6.25\%). Drug resistance was common among Gram negative isolates in three cases (50\%).

\section{CONCLUSION}

Streptococcus pneumoniae remains the most common aetiological agent of ABM in adults. Multidrug resistant Gram negative bacilli are also important emerging causes of ABM. This study shows the importance of centrifuged CSF Gram smear along with culture for the accurate diagnosis of ABM in developing countries. LAT can be used as a simple, rapid and convenient test to establish the bacterial aetiology in ABM.

\section{KEYWORDS}

Acute Bacterial Meningitis, Streptococcus Pneumoniae, Latex Agglutination Test.

HOW TO CITE THIS ARTICLE: Manjiyil IJ, Moorkoth AP, Santhakumari RD. Bacteriological profile of acute bacterial meningitis in adults in a tertiary care hospital in north Kerala. J. Evolution Med. Dent. Sci. 016;5(46):2884-2888, DOI: 10.14260/jemds/2016/672

\section{INTRODUCTION}

Acute Bacterial Meningitis (ABM) is a life-threatening infection with high rates of mortality and long-term neurological disability. Despite the availability of potent newer antibiotics, the mortality rate due to ABM remains significantly high in India and other developing countries (16-32\%). ${ }^{1}$ Any delay in the diagnosis and initiation of effective antimicrobial therapy can result in an unfavourable outcome.

Financial or Other, Competing Interest: None.

Submission 11-04-2016, Peer Review 19-05-2016,

Acceptance 24-05-2016, Published 08-06-2016.

Corresponding Author:

Dr. Irene Jose Manjiyil,

Assistant Professor,

Department of Microbiology,

Government Medical College

Thrissur, Kerala.

E-mail: drireneantony@gmail.com

DOI: $10.14260 /$ jemds $/ 2016 / 672$
As the clinical manifestations cannot always be relied upon, rapid and reliable diagnostic methods are of utmost importance for the prompt initiation of antimicrobial therapy. Microbiology laboratories play a critical role not only in the early identification of the causative bacterium and its antibiotic susceptibility pattern, but also in providing valuable information regarding the pathogens predominant in that area and the choice of antibiotic for empiric treatment. Increased awareness, availability and usage of vaccines can reflect a change in the epidemiological pattern of the pathogens responsible for ABM. Furthermore, for this potentially fatal infection the need for a periodic assessment of the continuing susceptibility of the causative bacteria to the antibiotic in use is necessary. This study was done to determine the bacterial pathogens responsible for $\mathrm{ABM}$ in adults and study their antibiotic susceptibility pattern 


\section{MATERIALS AND METHODS}

The study was carried out on 165 clinically suspected cases of ABM in adults admitted at Government Medical College, Kozhikode, for a period of one year from January 2009 to January 2010. The study was approved by our Institutional Ethics Committee. Criteria used for a definitive diagnosis of $\mathrm{ABM}$ in our study included a positive CSF culture with/without a positive Gram stain and/or a positive LAT along with at least one of the classical clinical manifestations of ABM and any one of the features of meningeal inflammation: a) CSF cell count $>10$ cells $/ \mathrm{mm}^{3}$ with predominant polymorphonuclear neutrophils, b) CSF protein $>45 \mathrm{mg} \%$, and c) CSF glucose $<40$ $\mathrm{mg} \%$.

Cases of post-traumatic and post-neurosurgical meningitis with an acute presentation were also included in this study group. A second episode of meningitis was considered a recurrence if it was due to a different organism from the first or if it was due to the same organism, but occurred more than three weeks after the completion of therapy for the initial episode. ${ }^{2}$ Information regarding vaccination against meningeal pathogens was obtained from the patients.

All CSF samples were collected aseptically in a dry sterile container and processed in the Microbiology Laboratory immediately; $2 \mathrm{~mL}$ of CSF was aliquoted into a sterile test tube and was subjected to centrifugation at $1000 \mathrm{rpm}$ for $10-15$ minutes. The deposit was used for culture and Gram staining. All CSF samples were inoculated on 5\% sheep blood agar with staphylococcal touch colony, chocolate agar, MacConkey's agar and brain-heart infusion broth for enrichment from which subcultures were done. Blood agar with staphylococcal touch colony and chocolate agar were kept in a candle jar with $10 \%$ $\mathrm{CO}_{2}$ and all media were incubated at $37^{\circ} \mathrm{C}$. The culture plates were inspected for the presence of growth after overnight incubation. When growth appeared, a secondary smear was performed from the colony. When no growth occurred, all the plates were incubated for a minimum period of 48 hours.

The isolates were identified by standard microbiological techniques and antibiotic susceptibility pattern was determined by Kirby-Bauer disc diffusion method on appropriate media in accordance with Clinical Laboratories Standards Institute (CLSI) guidelines. When organisms were present in the Gram smear, a direct antibiotic sensitivity testing was done. For Gram positive organisms, the following antibiotics were tested: Penicillin (10 units), Oxacillin $(1 \mu \mathrm{g})$, Cefoxitin $(30 \mu \mathrm{g})$, Ceftriaxone $(30 \mu \mathrm{g})$ and Vancomycin $(30 \mu \mathrm{g})$. The antibiotics tested for Gram negative bacilli were Gentamicin $(10 \mu \mathrm{g})$, Amikacin $(30 \mu \mathrm{g})$, Ceftriaxone $(30 \mu \mathrm{g})$, Ceftazidime $(30 \mu \mathrm{g})$, Piperacillin $(100 \mu \mathrm{g})$, Piperacillintazobactam $(100 \mu \mathrm{g} / 10 \mu \mathrm{g})$ and Meropenem $(10 \mu \mathrm{g})$.

The CSF supernatant was drawn off aseptically and used for detection of soluble antigens of Streptococcus pneumoniae; Haemophilus influenzae type b; Neisseria meningitidis A, B, C, Y and W135; E. coli K1 and Group B streptococcus by LAT using commercially available kits (Wellcogen Bacterial Antigen Kit, Remel Europe Ltd, U. K.). The procedure was performed as per the manufacture's guidelines.

\section{RESULTS}

During the one-year study period, a total of 165 CSF samples were collected and processed from clinically suspected cases of ABM. The bacterial pathogen was isolated by culture in 16 cases $(9.7 \%)$. All these 16 culture confirmed cases belonged to
16-55 years of age (mean age 37.15 years). Of these, 12 (75\%) were males and four (25\%) were females. CSF cell count varied from $150-11,600$ cells $/ \mathrm{mm}^{3}$ with a predominant neutrophilic pleocytosis. All culture proven cases had an elevated CSF protein above $45 \mathrm{mg} \%$ and $14(87.5 \%)$ cases had CSF protein above $200 \mathrm{mg} \%$. All 16 (100\%) cases had CSF glucose less than $40 \mathrm{mg} \%$.

Streptococcus pneumoniae was the predominant pathogen isolated in 7 cases $(43.8 \%$ of total culture positives) followed by Pseudomonas aeruginosa in 4 cases (25\%), Acinetobacter baumannii and Coagulase Negative Staphylococci (CoNS) in 2 cases each (12.5\%) and Staphylococcus aureus in one case (6.25\%) (Table 1). The rest of the CSF samples were sterile on routine bacterial culture. The distribution of cases is given in Figure 1. Streptococcus pneumoniae was isolated in 3 cases $(18.8 \%)$ of recurrent meningitis, which followed remote accidental head trauma. Only one patient had been vaccinated with a single dose of Pneumococcal Polysaccharide Vaccine 23 (PPSV23/Pneumovax).

16 culture positive cases $(9.7 \%)$ were also Gram smear positive. Additional 5 cases which included 4 cases of culture negative pneumococcal meningitis and 1 case of culture negative Gram negative bacillary meningitis could be detected by their typical morphology in the centrifuged Gram smear (Table 2 and 3). Thus Gram smear were positive in 21 samples (12.7\%), though pus cells could be detected in CSF by Gram stain in 35 samples (21.2\%) (See Table 2 and 3). Out of 165 samples, 130 samples (78\%) had no finding on Gram stain, culture or latex agglutination test. LAT could detect the capsular antigen of Streptococcus pneumoniae in 9 CSF samples (5.4\%), which included six culture positive and three culture negative cases. Out of the seven culture positive cases of Streptococcus pneumoniae, one was negative by LAT. Among 4 cases of Streptococcus pneumoniae which were culture negative, 3 were positive by LAT. No bacteria other than Streptococcus pneumoniae could be detected by LAT. Thus the aetiological agent could be detected in 19 cases $(11.5 \%)$ by Gram stain and culture and/or LAT.

All Streptococcus pneumoniae isolates were susceptible to all the antibiotics tested. No Penicillin resistance was detected among the pneumococcal isolates in this study. CoNS isolated in both cases were Methicillin resistant. No Methicillin resistance was detected for Staphylococcus aureus; $50 \%$ of Pseudomonas aeruginosa and Acinetobacter baumannii isolates were multi-drug resistant. All of the Gram negative isolates were sensitive to Meropenem. The antimicrobial susceptibility pattern of the isolates have been summarized in Table 4 . Among the 16 culture confirmed cases, the mortality rate was $43.8 \%$.

\begin{tabular}{|c|c|}
\hline Organism & Number (\%) \\
\hline Streptococcus pneumoniae & $7(43.8)$ \\
\hline Pseudomonas aeruginosa & $4(25)$ \\
\hline Acinetobacter baumannii & $2(12.5)$ \\
\hline Coagulase negative staphylococcus (CoNS) & $2(12.5)$ \\
\hline Staphylococcus aureus & $1(6.25)$ \\
\hline Total & $\mathbf{1 6}(100)$ \\
\hline Table 1: Bacterial Agents Isolated by CSF Culture \\
\hline
\end{tabular}




\begin{tabular}{|c|c|c|c|c|}
\hline \multirow{2}{*}{ Gram Smear } & \multirow{2}{*}{ No. } & \multicolumn{3}{|c|}{ Culture } \\
\hline & & Isolate & No. & $\%$ \\
\hline $\begin{array}{l}\text { Pus cells with } \\
\text { Gram positive } \\
\text { diplococci }\end{array}$ & 11 & $\begin{array}{l}\text { Streptococcus } \\
\text { pneumoniae }\end{array}$ & 7 & 63.6 \\
\hline \multirow{2}{*}{$\begin{array}{c}\text { Pus cells with } \\
\text { Gram positive } \\
\text { cocci } \\
\text { predominantly in } \\
\text { clusters }\end{array}$} & \multirow[t]{2}{*}{3} & $\begin{array}{c}\text { Coagulase } \\
\text { negative } \\
\text { staphylococci }\end{array}$ & 2 & 66.6 \\
\hline & & $\begin{array}{c}\text { Staphylococcus } \\
\text { aureus }\end{array}$ & 1 & 33.3 \\
\hline \multirow{2}{*}{$\begin{array}{l}\text { Pus cells with } \\
\text { Gram negative } \\
\text { bacilli }\end{array}$} & \multirow{2}{*}{7} & $\begin{array}{c}\text { Pseudomonas } \\
\text { aeruginosa }\end{array}$ & 4 & 57.1 \\
\hline & & $\begin{array}{l}\text { Acinetobacter } \\
\text { baumannii }\end{array}$ & 2 & 28.6 \\
\hline Total & 21 & & 16 & 45.7 \\
\hline
\end{tabular}

\begin{tabular}{|c|c|l|l|l|}
\hline Pus cells only & 14 & & - & \\
\hline $\begin{array}{c}\text { No pus cells, no } \\
\text { bacteria }\end{array}$ & 130 & & - & \\
\hline Total & 165 & & & \\
\hline Table 2: CSF Gram Smear and Culture Isolates (n = 165) \\
\hline
\end{tabular}

\begin{tabular}{|c|c|c|c|}
\hline & $\begin{array}{c}\text { Culture } \\
\text { Positive }\end{array}$ & $\begin{array}{c}\text { Culture } \\
\text { Negative }\end{array}$ & Total \\
\hline Smear positive & 16 & 5 & $21(12.7 \%)$ \\
\hline Smear negative & 0 & 144 & $\begin{array}{c}144 \\
(87.3 \%)\end{array}$ \\
\hline Total & $\begin{array}{c}\mathbf{1 6} \\
(\mathbf{9 . 7 \% )}\end{array}$ & $\begin{array}{c}\mathbf{1 4 9} \\
(\mathbf{9 0 . 3} \%)\end{array}$ & $\begin{array}{c}\mathbf{1 6 5} \\
(\mathbf{1 0 0 \% )}\end{array}$ \\
\hline \multicolumn{4}{|c|}{ Table 3: Correlation of CSF Gram Stain } \\
with Culture (n= 165)
\end{tabular}

\begin{tabular}{|c|c|c|c|c|c|}
\hline \multirow{2}{*}{ Antibiotics Tested } & \multicolumn{5}{|c|}{ Isolate, Number Sensitive (\%) } \\
\cline { 2 - 6 } & S. Pneumoniae & P. Aeruginosa & A. Baumannii & CoNS & S. Aureus \\
\hline Penicillin & $7(100)$ & NT & NT & 00 & 00 \\
\hline Oxacillin & $7(100)$ & NT & NT & 00 & $1(100)$ \\
\hline Cefoxitin & NT & NT & 00 & NT & NT \\
\hline Gentamicin & NT & 00 & $1(50)$ & NT & NT \\
\hline Amikacin & NT & $2(50)$ & $1(50)$ & NT & NT \\
\hline Piperacillin & NT & $2(50)$ & $1(50)$ & $2(100)$ & $1(100)$ \\
\hline Ceftriaxone & $7(100)$ & NT & $1(50)$ & NT & NT \\
\hline Ceftazidime & NT & NT & $2(100)$ & $1(100)$ \\
\hline Vancomycin & $7(100)$ & $2(50))$ & $1(50)$ & NT & NT \\
\hline Piperacillin-tazobactam & NT & $4(100)$ & $2(100)$ & NT & NT \\
\hline Meropenem & NT & \multicolumn{5}{l}{} \\
\hline
\end{tabular}

NT: Not Tested

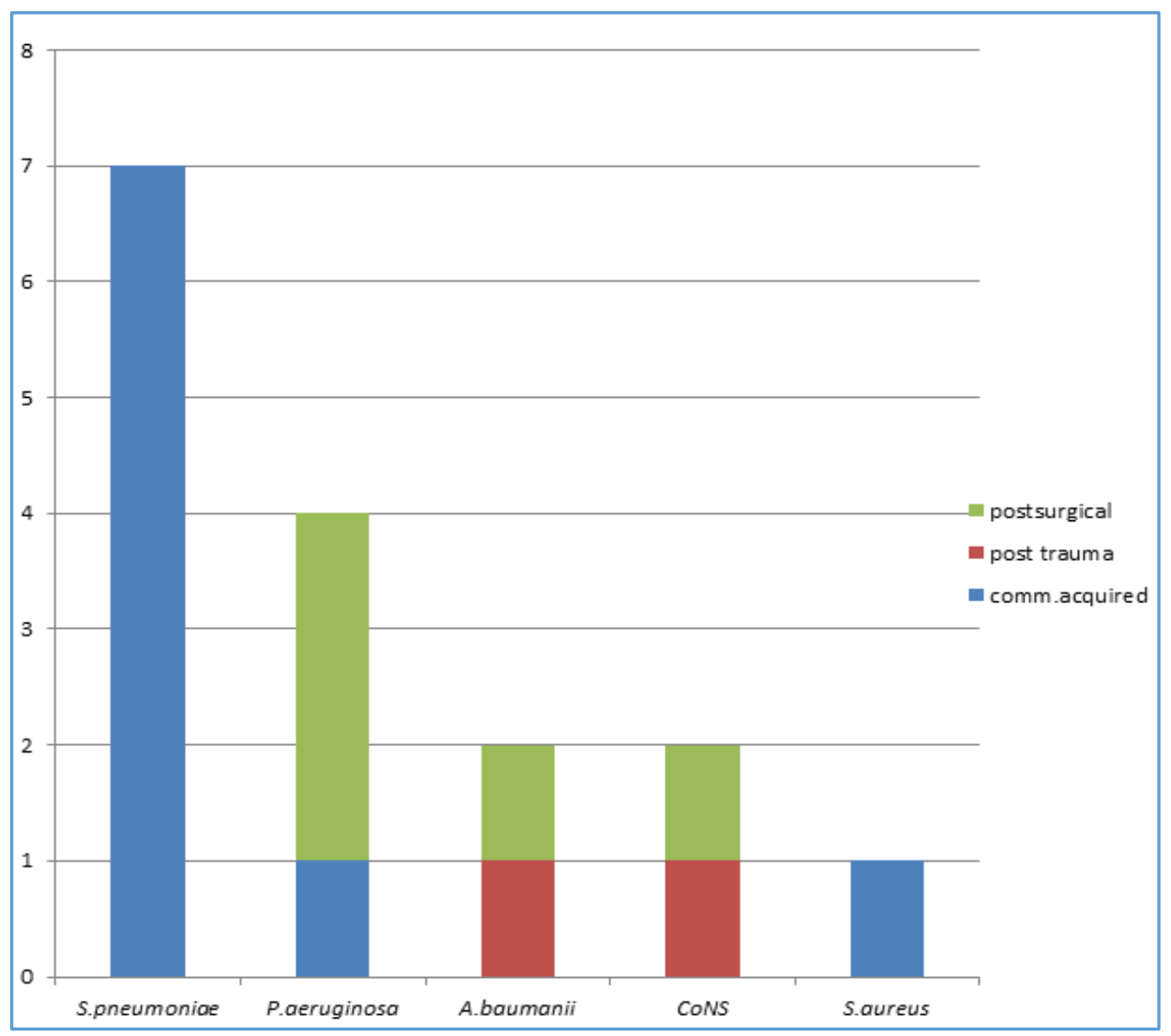

$\mathrm{X}$ axis - Organisms isolated, $\mathrm{Y}$ axis - No. of isolates

Fig. 1: Distribution of Culture Positive Cases 


\section{DISCUSSION}

Acute bacterial meningitis is a medical emergency and immediate steps should be taken to establish the specific cause and initiate effective therapy. The choice of antimicrobial therapy depends largely on the most common pathogen encountered in that particular area, antibiotic susceptibility pattern and the age of the patient.

In our study, Streptococcus pneumoniae remains the major aetiological agent of ABM in adults. Streptococcus pneumoniae was the most common aetiological agent of community-acquired meningitis accounting for $61.8 \%$ of cases in all age groups in a 10-year retrospective study from 19962005 in a Tertiary Neurocare Centre in South India. ${ }^{1}$ A similar trend has been reported in $66 \%$ of culture positive cases of meningitis in adults by Madhumita et al. ${ }^{3}$ Streptococcus pneumoniae was isolated from 3 (18.8\%) cases of communityacquired recurrent meningitis in the present study. Marc et al, has reported Streptococcus pneumoniae in $56.6 \%$ of culture confirmed episodes of recurrent bacterial meningitis associated with previous head injury. ${ }^{4}$ The predominance of Streptococcus pneumoniae as a cause of community-acquired recurrent meningitis following remote head injury and CSF leak has been reported by other authors. ${ }^{2,5}$

In our study, we report recurrent episode of pneumococcal meningitis in an already vaccinated patient. Our patient with third episode of pneumococcal meningitis within a period of five years had received a single dose of Pneumococcal Polysaccharide Vaccine 23 (PPSV23/Pneumovax) 3 years before the episode. In this case, infection might have been occurred with a different serotype not contained in the vaccine or the organism might have bypassed the circulating serum antibodies by directly invading the meninges. Cases of recurrent pneumococcal meningitis even after vaccination have been described in other studies. ${ }^{6}$ Studies reveal that of the serotypes isolated from the CSF of patients with pneumococcal meningitis, 74- 90\% represented those serotypes contained in PPSV23.7 Although, this vaccine is recommended for the prevention of bacteremic pneumococcal disease in high-risk groups, its efficacy in preventing pneumococcal meningitis has never been proven. ${ }^{7}$

No Penicillin resistance was detected among the pneumococcal isolates in this study. Resistance to Penicillin among Streptococcus pneumoniae isolated in India appears to be low, though it has been reported to vary between 1 to $12 \%$ according to some studies. ${ }^{8,9}$ However, resistant strains may create problems in treating the patients in the future.

Pseudomonas aeruginosa was isolated from 4 cases (25\%), among which 3 had undergone neurosurgical procedurescraniotomy with repair of dura, ventriculo-peritoneal shunt and repair of aneurysm rupture and one had severe underlying immunocompromised condition. Acinetobacter baumannii isolated from two cases $(12.5 \%)$ included one with intracerebral haemorrhage following accidental head trauma and other following craniotomy for brain tumour excision. CoNS was isolated from two cases (12.5\%), one who underwent ventriculo-peritoneal shunt and other with traumatic skull fracture following a road traffic accident. Staphylococcus aureus was isolated from a diabetic patient. (See Figure 1). These isolates were confirmed as aetiological agents by a repeat CSF culture.

Our findings was also in accordance with Chang et al, who reported Acinetobacter spp. (11.5\%), CoNS (11\%) and
Pseudomonas spp. (6\%) as important pathogens causing postneurosurgical meningitis in adults. ${ }^{10}$ There are some published case reports of early presentation of Gram negative bacillary meningitis in adults with accidental and neurosurgical trauma. The interval between the accident/neurosurgical procedures and the onset of meningitis in these cases has been reported as early from 2 days of the accident/surgical procedure.11,12 Staphylococcus aureus meningitis may be acquired in the community setting, associated with predisposing conditions such as diabetes mellitus and endocarditis.3,13 No Methicillin resistant Staphylococcus aureus was reported in the present study, though it has been reported by some authors. ${ }^{14}$

The overall mortality rate of culture positive cases in the study was $43.8 \%$. All our patients with pneumococcal meningitis survived, except one with diabetic mellitus who succumbed despite treatment with Ceftriaxone and Vancomycin; $50 \%$ of the Gram negative isolates were multidrug resistant. No Meropenem resistance was detected among Gram negative isolates. Antibiotic options for these multi-drug resistant strains were restricted to Meropenem. Our patients with Pseudomonas and Acinetobacter meningitis with serious co-morbidities had a fatal outcome despite receiving appropriate antibiotics and achieving CSF sterilization.

The cause of high mortality rate may be multiple drug resistance, neurosurgical complications and underlying immunocompromised state. Pseudomonas aeruginosa meningitis in adults with high mortality rate has been reported from post-neurosurgical cases and with underlying medical conditions by Huang et al and Taneja et al.15,16 Suzan Sacar et al, has reported post-surgical meningitis due to multi-drug resistant Acinetobacter baumannii which was successfully treated with high-dose Meropenem. ${ }^{17}$ All patients with staphylococcal meningitis were treated with Vancomycin and had a favourable outcome. Ceftriaxone may be considered an effective drug for the empirical treatment of ABM in adults.

Though the primary pathogens associated with ABM are Streptococcus pneumoniae, Haemophilus influenzae and Neisseria meningitidis, these aetiological agents and their relative frequencies may vary in different geographical areas. ${ }^{1}$ As compared to that which is seen in western studies, the relative incidence of meningitis caused by Haemophilus influenzae and Neisseria meningitidis is less in South East Asia.1,14 No cases of Haemophilus influenzae or meningococcal meningitis were detected by Gram stain, culture or LAT in our study. As ours being a tertiary care hospital, majority of our patients were referred from nearby hospitals and were already on antibiotic therapy before presentation. This could be the main reason for a low percentage of bacterial isolates on culture in our study. CSF sterilization might have occurred more rapidly with parenteral antimicrobial therapy. Moreover, our study being conducted only for a period of one year, a study for a longer period of time may help to detect these meningeal pathogens.

Several studies from India report culture negative cases of meningitis or a low CSF culture positivity, ranging from 6$50 \% .^{3}$ Only $1 \%$ of meningococcal meningitis cases have been reported in adults by Mani et al, in a ten-year retrospective study in a Tertiary Neurocare Centre in South India and according to them meningococcal meningitis has a low prevalence except during epidemics. ${ }^{1}$ Haemophilus influenzae type B meningitis has not been reported in the adult patients 
by Mani et al and claimed that the incidence has remained low for the past several decades. ${ }^{1}$ Only one case of communityacquired Haemophilus influenzae meningitis has been reported in adults during a period of 20 years by Bodin et al. ${ }^{18}$

Several newer molecular techniques for detecting bacterial DNA in CSF by Polymerase Chain Reaction (PCR) with a high sensitivity and specificity have emerged as powerful tools in the diagnosis of patients with culture negative meningitis. Unfortunately, PCR was not available in our hospital during the study period because of economic limitations.

Gram staining is the single most useful test for identifying $\mathrm{ABM}$, as it revealed more positive cases than cultures. ${ }^{14}$ In our study, Gram stain provided evidence of causative bacteria in 21 CSF samples (12.5\%). Of these, 16 samples yielded growth on culture and 5 were culture negative. It needs to be reiterated that simple tests like centrifuged Gram smear can help to establish the crucial diagnosis of ABM in our setup, especially in culture negative cases. Mani et al, has attributed their high yield of pathogens on Gram stain to their routine use of cytospin to concentrate the smear. ${ }^{1}$

A properly interpreted LAT can be used as a simple, rapid procedure suitable to be used as an adjunct laboratory test in patients pre-treated with antimicrobial therapy and whose Gram stain and CSF culture are negative. The false-negative LAT in case of a culture positive pneumococcal meningitis could possibly be due to very low antigen titres in CSF. It is also possible that the antiserum used in the diagnostic LAT kits does not detect all the capsular serotypes prevalent in a particular geographical area or probably as yet unrecognized serotypes are the causative agents. ${ }^{1}$ As LAT is mainly used to detect the specific pathogens of community-acquired meningitis, CSF samples which yielded organisms other than Streptococcus pneumoniae on culture gave a negative LAT test. A negative bacterial antigen test does not rule out infection caused by a specific meningeal pathogen. As the high costs of LAT kits is a drawback for its routine use in our laboratories, affordable indigenous LAT kits can help to detect the serotypes prevalent in our geographical area. ${ }^{1}$ To increase the cost effectiveness in a resource poor setting, LAT for pneumococcal antigen should be performed first, as it is the most common pathogen causing ABM in adults. ${ }^{1}$

\section{LIMITATIONS OF THE STUDY}

Being a tertiary care centre, most of the patients referred to our hospital were already on antimicrobial therapy. Minimum Inhibitory Concentration (MIC) of the antibiotic could not be done for the isolates.

\section{CONCLUSION}

Streptococcus pneumoniae still remains the major aetiological agent of $\mathrm{ABM}$ in adults. Multi-drug resistant Gram negative bacilli are also important emerging causes of ABM. This study highlights the importance of centrifuged CSF Gram smear for accurate and early diagnosis of ABM. LAT can be used as a simple, rapid and convenient test to establish the bacterial aetiology in ABM.

\section{REFERENCES}

1. Mani R, Pradhan S, Nagarathna S, et al. Bacteriological profile of community-acquired acute bacterial meningitis: a ten year retrospective study in a tertiary neurocare centre in South India. Indian J Med Microbiol 2007;25(2):108-14.
2. Marlene LD, Stephen B Calderwood, David J Weber, et al. Acute bacterial meningitis in adults-a review of 493 episodes. N Engl J Med 1993;328:21-8.

3. Madhumita P, Gupta N. Clinical and bacteriological spectrum of community-acquired acute bacterial meningitis in adults at a tertiary care hospital in northern India. International Journal of Nutrition, Pharmacology, Neurological Diseases 2011;1(2):194200.

4. Marc T, Nigel C. Epidemiology, aetiology, pathogenesis, and diagnosis of recurrent bacterial meningitis. Clin Microbiol Rev 2008;21(3):519-37.

5. Kirsten SA, Diederik Van de Beek, Matthijs CB, et al. Community-acquired recurrent bacterial meningitis in adults. Clin Infect Dis 2007;45(5):46-51.

6. Paudyal BP. Twelve episodes of meningitis in the same patient. Kathmandu University Medical Journal 2007;5(2):243-6.

7. Matthijs CB, Allan RT, Diederik van de Beek. Epidemiology, diagnosis, and antimicrobial treatment of acute bacterial meningitis. Clin Microbiol Rev 2010;23(3):467-92.

8. Deva A, Prasad SR, Madappa BP, et al. Pneumococcal infections at a rural tertiary care hospital: a seven year study on isolation rate, clinical spectrum and antibiogram. J Clin Diagn Res 2014;8(2):50-2.

9. Kanungo R, D' Lima D, Rajalakshmi B, et al. Emerging antibiotic resistant pneumococci in invasive infections in South India: need for monitoring. Indian J Pharmacol 2002;34:38-43.

10. Chang WN, Lu CH, Huang CR, et al. Changing epidemiology of adult bacterial meningitis in Southern Taiwan: a hospital-based study. Infection 2008;36(1):15-22.

11. Fahmi YK, Mohammed A, Deshmukh A. Nosocomial Escherichia coli meningitis in adults: report of four cases and literature review. J Neurosci Rural Pract 2013;4(3):349-51.

12. Tung YL, Wen JC, Ming KH, et al. Post-operative meningitis after spinal surgery: a review of 21 cases from 20,178 patients. BMC Infectious Diseases 2014;14:220.

13. Matthijs CB, Keizerweerd GD, De Gans J, et al. Community-acquired Staphylococcus aureus meningitis in adults. Scand J Infect Dis 2009;41(5):375-7.

14. Modi GB, Patel KD, Soni ST, et al. Bacteriological profile of pyogenic meningitis in tertiary care hospital, Ahmedabad. Natl J Med Res 2012;2(3):313-7.

15. Huang CR, Lu CH, Chuang YC, et al. Adult pseudomonas aeruginosa meningitis: high incidence of underlying medical and /or post-neurosurgical conditions and high mortality rate. Jpn J Infect Dis 2007;60(6):397-9.

16. Taneja J, Mishra B, Thakur A, et al. Pseudomonas aeruginosa meningitis in post neurosurgical patients. Neurology Asia 2009;14(2):95-100.

17. Saçar S, Turgut H, Cenqer DH, et al. Successful treatment of multi-drug resistant. A baumannii meningitis. J Infect Dev Ctries 2007;1:342-4.

18. Bodin K, Pantip C, Alan G. Acute bacterial meningitis in adults: a 20 year review. Southeast Asian J Trop Med Public Health 2004;35(4):886-92. 